\title{
The SHiP facility at CERN
}

\author{
Giovanni De Lellis ${ }^{1, a}$ \\ ${ }^{1}$ Università "Federico II" and INFN, Naples, Italy
}

\begin{abstract}
Searches for new physics with accelerators are being performed at the LHC, looking for high massive particles coupled to matter with ordinary strength. A new experimental facility meant to search for very weakly coupled particles in the few $\mathrm{GeV}$ mass domain has been recently proposed. The existence of such particles, foreseen in different theoretical models beyond the Standard Model, is largely unexplored from the experimental point of view. A beam dump facility, built at CERN in the north area, using $400 \mathrm{GeV}$ protons is a copious factory of charmed hadrons and could be used to probe the existence of such particles. The beam dump is also an ideal source of tau neutrinos, the less known particle in the Standard Model. In particular, tau anti-neutrinos have not been directly observed so far. We report the physics potential of such an experiment and outline the performances of a detector operating at the same facility for the search for the $\tau \rightarrow \mu \mu \mu$ decay.
\end{abstract}

\section{Introduction}

The discovery of the Higgs boson [1, 2] is certainly a big triumph of the Standard Model. In particular, given its measured mass of $125.5 \pm 0.5 \mathrm{GeV}$, it could well be that the Standard Model is an effective theory working all the way up to the Planck scale. Indeed, the $\lambda$ coupling of the quartic Higgs potential keeps the same sign making the vacuum stable on the time scale of the Universe [3] and its running value stays finite up to very high energy scales [4]. Nevertheless, there are several facts deserving an explanation that the Standard Model is unable to provide: the existence of dark matter and its nature, the baryonic asymmetry of the Universe and neutrino masses. It is therefore clear the new physics is there and presumably several new particles have still to be discovered.

Searches for new physics with accelerators are being carried out extensively at the LHC, that is especially suited to look for high mass particles with ordinary couplings to matter. Complementary searches for very weakly coupled and therefore long lived particles require a beam dump facility. Such a facility is made of a high density proton target, followed by a hadron stopper and a muon shield. Apart from residual muons, the only remaining particles are electron, muon and tau neutrinos on top of hidden, long lived particles produced either in proton interactions or in secondary particle decays.

A new experiment, Search for Hidden Particles (SHiP), has been proposed [5], designed to operate at a beam dump facility to be built at CERN and to search for weakly coupled particles in the few $\mathrm{GeV}$ mass range. Since a high intensity tau neutrino flux is produced by such a facility from $D_{s}$ decays, the experimental apparatus fore-

\footnotetext{
${ }^{\mathrm{a}}$ On behalf of the SHiP Collaboration, e-mail: giovanni.de.lellis@ cern.ch
}

sees a neutrino detector to study the tau neutrino crosssection and discover the tau anti-neutrino. The physics case for such an experiment is widely discussed in Ref. [6]. We will review here the physics potential of this experiment for a selection of physics channels and we also give the performances of a dedicated experiment searching for $\tau \rightarrow \mu \mu \mu$ decays, operating at the same beam dump Facility at CERN.

\section{The SHiP facility and apparatus}

The facility will integrate in five years $2 \times 10^{20} 400 \mathrm{GeV}$ protons, produced by the SPS accelerator complex, impinging on a 10 interaction length target made of Molybdenum and Tungsten, followed by an hadron absorber. Hidden particles in the $\mathrm{GeV}$ mass range would be produced mostly by the decay of charmed hadrons produced in proton interactions. $D_{s}$ mesons, copiously produced among charmed hadrons, are a good source of tau neutrinos through their fully leptonic decay. Therefore, the SHiP facility is ideal also to study the physics of tau neutrinos, the less known particle in the Standard Model.

Figure 1 shows the SHiP facility to be placed in the North Area: downstream of the target, the hadron absorber filters out all hadrons, therefore only muons and neutrinos are left. An active muon shield is designed with two sections with opposite polarity to maximize the muon flux reduction: it reduces the muon flux from $\sim 10^{10}$ muons/spill down to about $10^{4}$ per spill. The slow extraction of $4 \times 10^{13}$ protons is foreseen in each spill, designed to be $1 \mathrm{~s}$ long to reduce the detector occupancy. A first successful test of the SPS cycle with a 1s long spill was performed in April 2015. The tau neutrino detector is located downstream of the muon shield, followed by the decay vessel and the detector for hidden particles. The Technical Design report of 


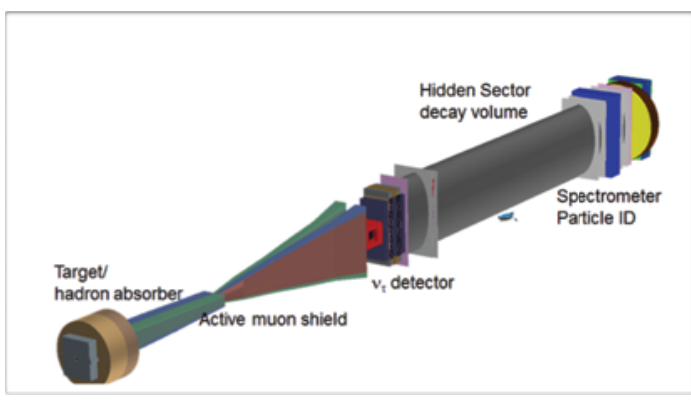

Figure 1. The beam dump facility and the SHiP detector.

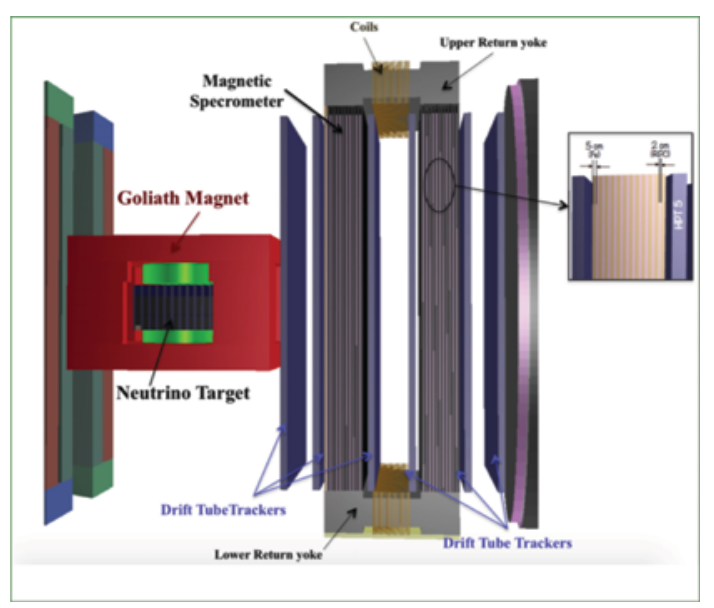

Figure 2. The neutrino detector of the SHiP apparatus.

the SHiP facility is expected to be ready in 2018, the construction and installation to last until the third long shutdown of the LHC and the data taking to start soon after in 2026.

The neutrino detector is made of a magnetised target region, followed by a muon spectrometer, as shown in Fig. 2. The neutrino target is based on the emulsion cloud chamber technology employed by the OPERA experiment [7], with a compact emulsion spectrometer, made of a sequence of very low density material and emulsion films to measure the charge and momentum of hadrons in magnetic field. Indeed, this feature would allow to discriminate between tau neutrinos and anti-neutrinos also in the hadronic decay channels of the tau lepton. The emulsion target is complemented by high resolution tracking chambers to provide the time stamp to the event and connect muon tracks from the target to the muon spectrometer.

The detector for hidden particles, shown in Fig. 3, is located in the downstream part a $60 \mathrm{~m}$ long evacuated decay vessel, with an elliptical transverse section of $5 \times 10 \mathrm{~m}^{2}$, with the longer axis vertically. The hidden particles are supposed to decay within the vessel. The requirement to have less than 1 neutrino interaction in the vessel over the full run sets the pressure to about $10^{-3}$ mbar. Downstream of the vessel, a magnetic spectrometer is located: it is made of straw tubes with a material budget of $0.5 \% X_{0}$ per station, achieving a position resolution of

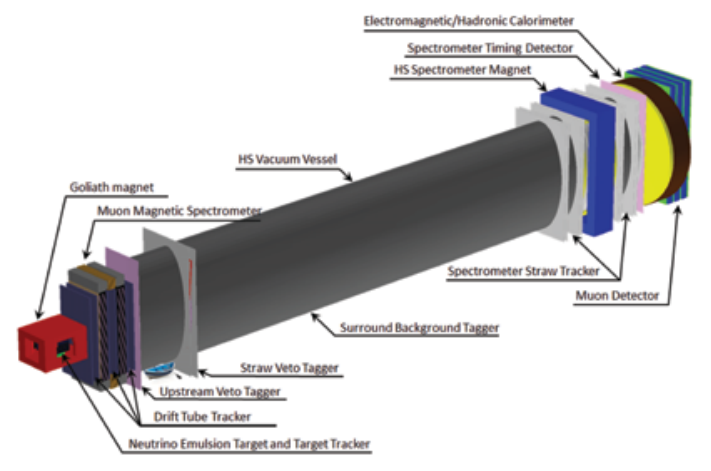

Figure 3. The SHiP detector: the detector for hidden particles is located downstream of the decay vessel.

$120 \mu \mathrm{m}$ per straw, with 8 hits per station on average. This gives a momentum resolution of about $1 \%$. The challenge is to build $5 \mathrm{~m}$ long straw tubes and the large vacuum vessel. The vessel would be sorrounded by a liquid scintillator layer to tag particles coming from outside. Downstream of the spectrometer, an hadronic and electromagnetic calorimeter and a muon filter are meant for particle identification. A timing detector with a 50ps resolution complements the detector to suppress the combinatorial background.

\section{Search for hidden particles}

Extensions of the Standard Model in the low mass region foresee the existence of particles as singlets with respect to the Standard Model gauge group. These particles couple to different singlet composite operators (so-called Portals) of the Standard Model. These particles might be fermions, like heavy neutral leptons, scalar particles coupled to the Higgs like the light inflaton, vector particles like the dark photon and eventually pseudoscalar particles like pseudoNambu-Goldstone bosons (or "axions"). The SHiP detector located immediately downstream of a beam dump has the potentiality to discover all of them, since it is sensitive to very weakly interacting and long lived particles in a wide unexplored range of their masses and couplings. We describe in the following its sensitivity to dark photons and heavy neutral leptons. For the sensitivity to the other portals, we refer to Ref. [5].

\subsection{Search for hidden photons}

Dark photons are vectorial particles coupled to the photon with a lagrangian term $L=-k B_{\mu \nu} V^{\mu \nu} / 2$. In a beam dump facility, they can be produced both in the proton bremsstrahlung and in the decay of particles like $\pi^{0}, \eta$, $\omega$ and $\eta^{\prime}$. If we assume that no lighter hidden particles exist, the dark photon decays into SM particles with final states like $e^{+} e^{-}, \mu^{+} \mu^{-}$or $q \bar{q}$. Recently, data from old experiments have been reanalysed and exclusion plots have been derived together with cosmological limits [8, 9]. Figure 4 shows the exclusion limits at $90 \%$ C.L. set by SHiP in case no signal is found. The parameters displayed are 


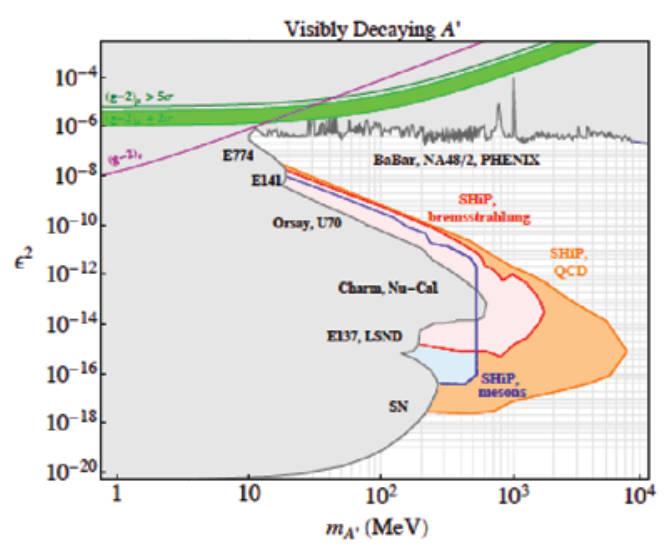

Figure 4. Exclusion limit set by the SHiP experiment in case no signal is found in the plane defined by its mass and $\epsilon^{2}$ squared coupling. Limits from other experiments are also drawn for comparison.

the mass and $\varepsilon^{2}$ squared coupling of the dark photon. The contributions from proton bremsstrahlung and meson decays are indicated: the sensitivity extends up to $10 \mathrm{GeV}$. It is worth noting that the region investigated by SHiP largely exceeds those explored by previous searches in the region of cosmological interest.

\subsection{Search for heavy neutral leptons}

Neutrino masses are usually explained by the see-saw mechanism with a lagrangian term given by

$$
L={ }_{\imath} \bar{N}_{I} \partial_{\mu} \gamma^{\mu} N_{I}-Y_{I \alpha} \bar{N}_{I}^{C} \tilde{H} L_{\alpha}-M_{I} \bar{N}_{I}^{C} N_{I}+\text { h.c. }
$$

where 3 right-handed heavy neutrinos $N_{I}$ are introduced. The mass of active neutrinos is given by $m_{v} \simeq m_{D}^{2} / M_{I}$ where $m_{D} \simeq Y_{I \alpha} v$ and $v \simeq 246 \mathrm{GeV}$ is the Higgs vacuum expectation value. A massive neutrino with $M_{I}$ of the order of $1 \mathrm{GeV}$ and Yukawa couplings $Y_{I \alpha}$ of the order of $10^{-7}$ would account for an active neutrino mass $m_{v} \simeq 0.05 \mathrm{eV}$, as measured by neutrino oscillation experiments.

If several conditions are satisfied, among which CP is not conserved, these right-handed neutrinos could also explain the leptogenesis and therein the baryogenesis [10, 11]. Such models do not pretend to explain also dark matter: a dark matter candidate could be provided by a fourth right-handed neutrino or an axion. Moreover they do not require two of the three heavy neutrinos to be quasidegenerate because $\mathrm{CP}$ violation does not need to be enhanced by mass degeneracy.

In the attempt of explaining also dark matter with three heavy neutrinos, it was first introduced in 2005 the so-called $v$ MSM $[12,13]$, later followed by refined versions [14]. The role of the three right-handed neutrinos is different as well as their mass: $M_{1}$ could be a dark matter candidate with the mass of several $\mathrm{keV}$ while $M_{2}$ and $M_{3}$ should be states with a mass of about $1 \mathrm{GeV}$ and quasidegenerate to provide the baryon asymmetry of the Universe. In order to be a dark matter candidate, $M_{1}$ has to

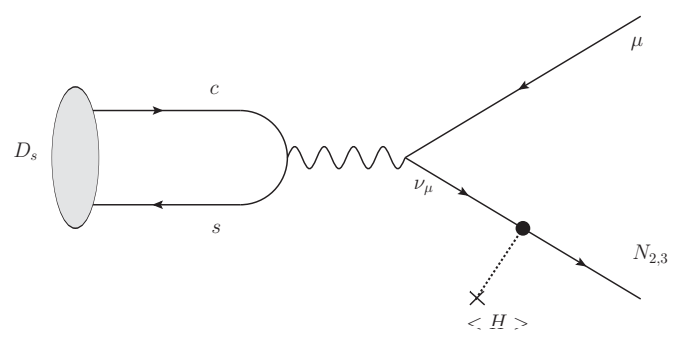

Figure 5. An example of a charmed hadron decay producing $N_{2,3}$ states.

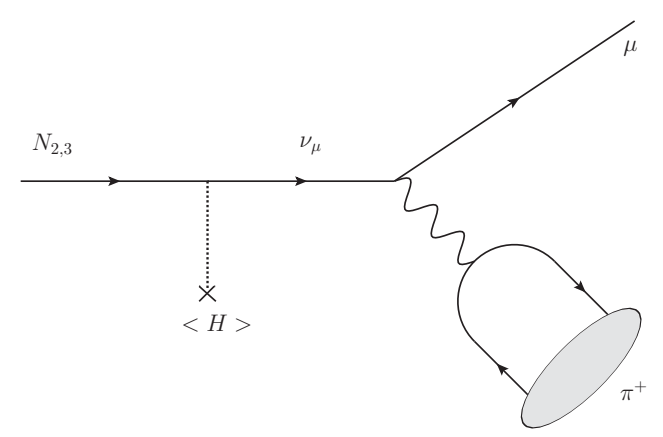

Figure 6. An example of the $N_{2,3}$ decay modes: $\pi$ and $\mu$ are produced in the final state.

be so light (several $\mathrm{KeV}$ ) that its only visible decay channel could be $N_{1} \rightarrow \gamma v$, producing a monochromatic line at $M_{I} / 2$ in the X-ray spectrum. A peak around $3.5 \mathrm{keV}$ was recently reported by two different groups analysing data of astronomical X-ray surveys $[15,16]$. The reported peak shows a low statistical significance and it will be studied by next generation space missions with sufficient energy resolution, $\Delta E / E \simeq 10^{-3}$.

The states $N_{2}$ and $N_{3}$ could be produced in the decay of sufficiently massive particles like charmed hadrons with a diagram shown, as an example, in figure 5. The states produced in this way would be long lived particles that in turn could decay into $\pi \mu$ as it is illustrated, as an example, in figure 6 . Therefore, by measuring the invariant mass of the $\pi \mu$ system, one would expect a peak to show up.

It is worth noticing that there are more cosmological than experimental constraints in the mass region around 1 $\mathrm{GeV}$. Charmed hadrons are the ideal parents of such heavy neutral leptons if their mass is around $1 \mathrm{GeV}$. Indeed, with kaon decays one is sensitive only to masses lower than 400 $\mathrm{MeV}$, while the cross-section for the production of beauty hadrons is a factor of 20 to 100 lower. Moreover, given that beauty hadrons mostly decay to charmed hadrons, the explored mass range would only extend from 2 to $3 \mathrm{GeV}$. A proton beam dump facility is the ideal place to copiously produce charmed hadrons and to search for heavy neutral leptons of this mass and lifetime $\left(10^{-5} \mathrm{~s}\right)$ range.

Figure 7 shows the experimental and cosmological bounds on the search for heavy neutral leptons. It also reports superimposed the exclusion limits at 90\% C.L. SHiP could set in case no signal is found. The top plot assumes 

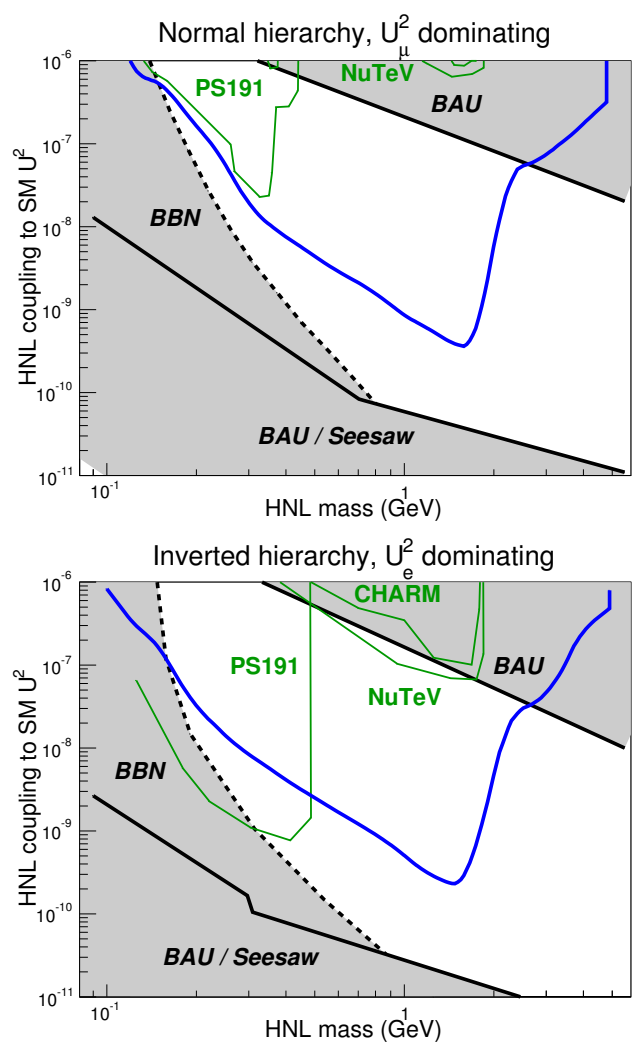

Figure 7. Exclusion limits at $90 \%$ C.L. sets by SHiP in case no signal is found. Top: normal hierarchy with $U_{\mu}^{2}$ dominating according to Ref. [17]. Bottom: inverted hierarchy with $U_{e}^{2}$ dominating according to Ref. [18].

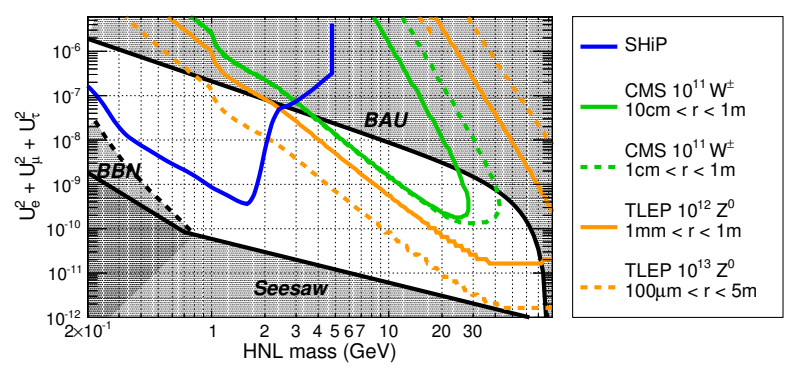

Figure 8. The region explored by the SHiP experiment is compared with cosmological constraints and the projected results of the CMS experiment and TLEP project.

the model reported in Ref. [17] with normal hierarchy and a dominant muon coupling, $U_{e}^{2}: U_{\mu}^{2}: U_{\tau}^{2}=1: 16: 3.8$, while the bottom plot refers to the model in Ref. [18] for inverted hierarchy and a dominant electron coupling, $U_{e}^{2}: U_{\mu}^{2}: U_{\tau}^{2}=48: 1: 1$. The parameter space explored by $\mathrm{SHiP}$ extends in the cosmologically relevant region that is experimentally unexplored. Moreover, the region explored by SHiP is complementary to the projected results of LHC projects at the end of their run and with future projects such as TLEP as it is shown in Figure 8.

\section{Physics with the neutrino detector}

The tau neutrino is the less known particle in the Standard Model. Four candidates were firstly reported in 2001 by the DONUT experiment [19] and the observation of this particle was finally confirmed in 2008 when 9 candidates events were reported with an estimated background of 1.5 [20]. In the same paper they reported, for the first time, the tau neutrino cross-section where the constant term was measured to be $\sigma_{v_{\tau}}^{\text {const }}=(0.39 \pm 0.13 \pm$ $0.13) \times 10^{-38} \mathrm{~cm}^{2} \mathrm{GeV}^{-1}$. The large uncertainty is due to the poor statistical sample and to the rather scarce knowledge of the incoming flux. On top of the large uncertainty, DONUT could not separate tau neutrinos from antineutrinos. Moreover, the OPERA experiment [7] has detected five tau neutrinos [21-25], discovering the tau neutrino appearance from muon neutrino oscillations. The only leptonic decay observed by OPERA [23] shows negative charge as expected from a $v_{\tau}$ interaction. Therefore, so far there is no direct evidence for tau anti-neutrinos.

The number of $v_{\tau}$ and $\bar{v}_{\tau}$ emerging from the molybdenum target can be estimated as:

$$
N_{v_{\tau}+\bar{\nu}_{\tau}}=4 N_{p} \frac{\sigma_{c \bar{c}}}{\sigma_{p N}} f_{D_{s}} \operatorname{Br}\left(D_{s} \rightarrow \tau\right)=5.7 \times 10^{15}
$$

where

- $N_{p}$ is the number of interacting protons (all incoming ones);

- $\sigma_{c \bar{c}}=18.1 \pm 1.7 \mu$ barn [26] is the associated charm production per nucleon;

- $\sigma_{p N}=10.7$ mbarn is the hadronic cross-section per nucleon in a Mo target. The inelastic cross-section $p A$ shows the $A^{0.71}$ dependence [27];

- $f_{D_{s}}=\left(7.7 \pm 0.6_{-0.4}^{+0.5}\right) \%$ [28] is the fraction of $D_{s}$ mesons produced;

- $\operatorname{Br}\left(D_{s} \rightarrow \tau\right)=(5.54 \pm 0.24) \%$ [29] is the $D_{s}$ branching ratio into $\tau$;

- the factor 4 accounts for the charm pair production and for the two $v_{\tau}$ produced per $D_{s}$ decay.

The SHiP facility is therefore a $v_{\tau}$ factory, with $5.7 \times$ $10^{15}$ tau neutrinos produced, equally divided in neutrinos and anti-neutrinos. The uncertainty on this number is about $14 \%$ but the uncertainty on the differential neutrino flux is estimated to be about $20 \%$.

Given the neutrino target mass of 9.6 tons, the neutrino flux, the geometrical acceptance of the detector and the standard model cross-section [30], one expects about 6700 interactions of tau neutrinos and 3400 of tau anti-neutrinos. An uncertainty smaller than $10 \%$ affects the cross-section mainly due to uncertainties on scale choices, parton distribution functions and target mass corrections [30]. Therefore the uncertainty on the expected number is dominated by the $20 \%$ error on the differential neutrino flux.

The charged-current $v_{\tau}\left(\bar{v}_{\tau}\right)$ differential cross-section is given by five structure functions. The contribution to the cross-section of $F_{4}$ and $F_{5}$ structure functions, introduced by Albright and Jarlskog [31], is negligible in muon 


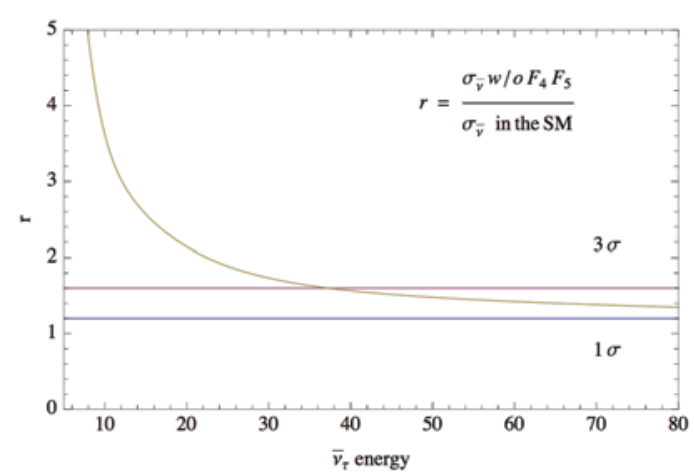

Figure 9. Energy dependence of the ratio between the DIS cross section in the $F_{4}=F_{5}=0$ hypothesis and the SM prediction for $\bar{v}_{\tau}$.

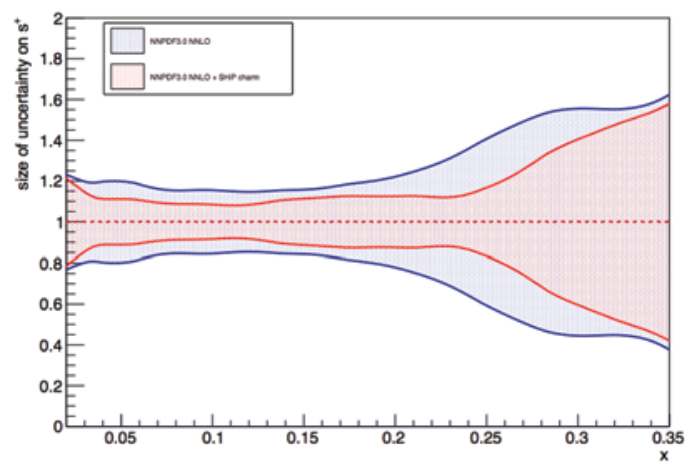

Figure 10. Improvement of the accuracy on $s^{+}$with SHiP (red) compared to the present status (blue) in the $0.02<x<0.35$ range.

and electron neutrino interactions due to the charged lepton mass. On the contrary, given the non-negligible mass of the $\tau$ lepton, tau neutrino scattering is sensitive to their contribution.

At the leading order, in the limit of massless quarks and target hadrons, the predicted values are $F_{4}=0$ and $2 x F_{5}=F_{2}$ [31]. Calculations at NLO show that $F_{4}$ gives a contribution to the cross-section of about $1 \%$ at the neutrino energy of $10 \mathrm{GeV}$ [30].

SHiP is sensitive to $F_{4}$ and $F_{5}$. Indeed, the hypothesis of $F_{4}=F_{5}=0$ would significantly increase the $v_{\tau}$ and $\bar{v}_{\tau}$ charged-current deep-inelastic cross sections and the corresponding number of expected $v_{\tau}$ and $\bar{v}_{\tau}$ interactions. Figure 9 shows the energy dependence of the ratio between the tau anti-neutrino cross-section when $F_{4}$ and $F_{5}$ vanish and the Standard Model prediction. The region below $40 \mathrm{GeV}$ is highly sensitive to the non-zero values of these structure functions: a $3 \sigma$ excess is expected in this region, given that the ratio exceeds 1.6.

\subsection{Strange parton distribution}

Charmed hadrons are produced in neutrino and antineutrino charged-current interactions at the level of about
5\%. Experiments based on calorimetric technology identify charmed hadrons only in their muonic decay channel, when two opposite sign muons are produced in the final state. A cut of $5 \mathrm{GeV}$ is applied to muons in order to suppress the background due to punch-through pions. The nuclear emulsion technology, instead, identifies topologically the charmed hadron by detecting its decay vertex. Energy cuts are therefore much looser, thus providing a better sensitivity to the charm quark mass. Moroever, a large statistical gain is provided by the use of hadronic decay modes [32]. Indeed, despite the fact that 1.280.000 $v_{\mu}$ and $270.000 \bar{v}_{\mu}$ charged-current interactions were collected by the NuTeV/CCFR Collaboration, only $5102 v_{\mu}$ and $1458 \bar{v}_{\mu}$ events were identified as charm production induced by neutrino charged-current interactions. The largest number of events in an emulsion experiment was collected by CHORUS which integrated 2013 charm candidates from $v_{\mu}$ and only 32 from $\bar{v}_{\mu}$ [33]. SHiP will integrate about $10^{5}$ charm candidates, more than one order of magnitude larger than the present statistics, with a large $(\sim 30 \%)$ contribution from anti-neutrinos. Charm production in neutrino scattering is extremely sensitive to the strange quark content of the nucleon, especially with anti-neutrinos where the $s$-quark is dominant. SHiP will improve significantly the uncertainty on the strange quark distribution in the nucleon as shown in Fig. 10 in terms of $s^{+}=s(x)+\bar{s}(x)$ in the $0.02<x<0.35$ range.

\section{A dedicated experiment to search for $\tau \rightarrow \mu \mu \mu$ decays}

The study of charged lepton flavour violation as a probe of new physics is expected to make a big step forward in the next few years with the MEG and MEG-II at PSI, with the proposed search of the $\mu \rightarrow$ eee decay at PSI and with the new searches for $\mu$ to $e$ conversion, Mu2e at FNAL and COMET at JPARC. Charged lepton flavour violation in $\tau$ decays is illustrated in Fig. 11. So far the best limits were achieved at the $B$ factories where about $10^{9} \tau$ leptons were collected. With an almost background free analysis, an upper limit $\operatorname{Br}(\tau \rightarrow \mu \mu \mu)<2.1 \times 10^{-8}$ was set at $90 \%$ C.L. [34]. The Belle-II experiment might bring it down to the level of $10^{-9}$ or even lower. LHCb has collected about $10^{11} \tau$ leptons and performed this search with an irreducible background from the $D_{s} \rightarrow \eta \mu \nu$ with subsequent $\eta \rightarrow \mu \mu$ decay: they have achieved a similar upper limit, $4.6 \times 10^{-8}$ [35], with an integrated luminosity of $1.0 \mathrm{fb}^{-1}$ of proton-proton collisions at a centre-of-mass energy of $7 \mathrm{TeV}$ and $2.0 \mathrm{fb}^{-1}$ at $8 \mathrm{TeV}$. There are prospects for $\mathrm{LHCb}$ to improve this limit when $50 \mathrm{fb}^{-1}$ will be integrated but they will hardly compete with $B$ factories.

$3 \times 10^{15} \tau$ leptons will be produced in the target of the SHiP Facility. Nevertheless, a thick $\left(10 \lambda_{\text {int }}\right)$ target is not suitable for this kind of search because the multiple Coulomb scattering would spoil the momentum resolution of the produced muons, thus spoiling the invariant mass resolution of the $3 \mu$ system, essential to identify the $\tau$. The physics case for a $\tau \rightarrow \mu \mu \mu$ dacay experiment at the SHiP Facility has been discussed in Ref. [6]. We revise the main 


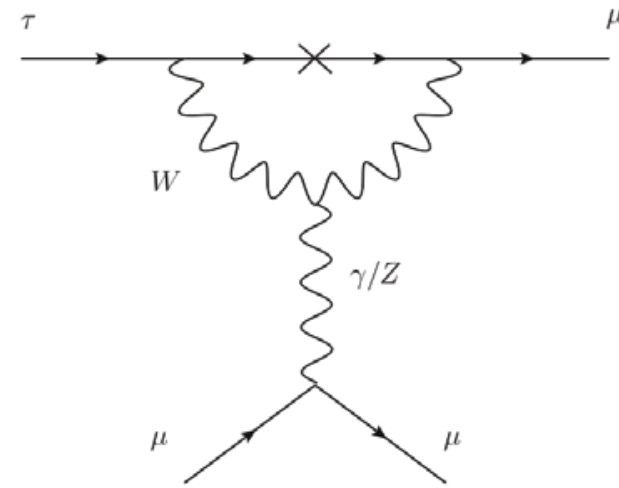

Figure 11. Feynman diagram of the $\tau \rightarrow \mu \mu \mu$ decay.

points of the discussion here, together with the coexistence of such a dedicated experiment with the SHiP detector.

We assume a $1 \mathrm{~mm}\left(0.01 \lambda_{\text {int }}\right)$ tungsten target where only $1 \%$ of the protons will interact and $8 \times 10^{12} \tau$ s will be produced. About $90 \%$ of the $\tau \mathrm{s}$ will decay outside of the target. Downstream of the target, a high precision tracking system will be placed in magnetic field: silicon pixel technology has the required resolution. This tracker, designed with a concept similar to the LHCb VELO [36], would be placed $8 \mathrm{~mm}$ far from the beam axis, leaving the remaining $99 \%$ of the protons to pass through and reach the SHiP target downstream. This expedient ensures the compatibility with the SHiP experiment. Downstream of the high resolution tracker, a magnetised hadron absorber will act as a muon identifaction system: the magnetic field will ensure that the curvature can be used to improve the track matching with the vertex detector. All three muons will get identified and measured. An optimisation of the material used and their size has to be done to tune the efficiency and mass resolution versus the particle misidentification. A precise timing detector will complete the experimental apparatus to reduce combinatorial background.

The geometrical acceptance of such a detector is $\varepsilon \sim$ $33 \%$, defined by the inner and outer muon chamber efficient in the $3<\vartheta<170 \mathrm{mrad}$ angular range. A cut on the muon momentum above $5 \mathrm{GeV}$ is also applied in order to have muons detected after passing the hadron absorber. Figure 12 shows the momentum distribution of muons originated from $\tau$ decays. The main background source is given by the $D_{s} \rightarrow \eta \mu \nu$ decay with subsequent $\eta \rightarrow \mu \mu \gamma$, which gives an irreducible background source. If the invariant mass of two muons is required to be larger than $450 \mathrm{MeV}$, a further reduction of this background is achieved, with about $1.5 \times 10^{5}$ remaining events. Accounting for the expected background and the number of detectable $\tau$ decays, a sensitivity to the branching ratio down to $10^{-10}$ would be achievable.

We therefore conclude that such an experiment could be carried out in parallel to the SHiP experiment and would be competitive in the future landscape of $\tau \rightarrow \mu \mu \mu$ searches.

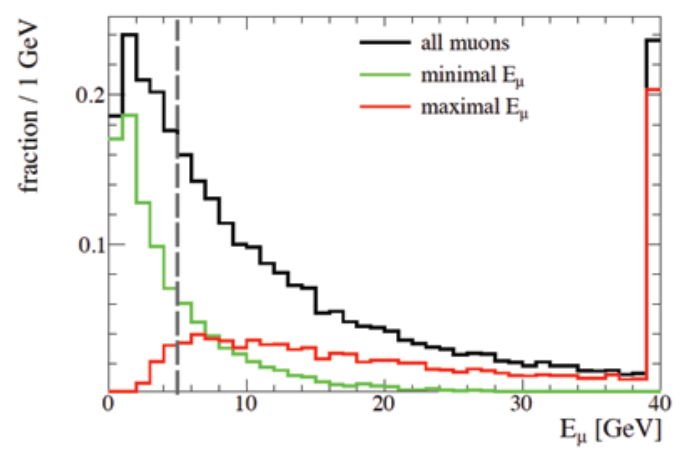

Figure 12. Momentum spectrum of muons produced from the $\tau$ decay.

\section{References}

[1] ATLAS Collaboration, Phys.Lett. B716, 1-29 (2012).

[2] CMS Collaboration, Phys.Lett. B716, 30-61 (2012).

[3] G. Degrassi et al., JHEP 1208, 098 (2012).

[4] S. Heinemeyer, Higgs Physics, arXiv:1405.3781.

[5] M. Anelli et al., CERN-SPSC-2015-016, SPSC-P350, arXiv:1504.04956.

[6] S. Alekhin et al., CERN-SPSC-2015-017, SPSCP350-ADD-1, arXiv:1504.04855.

[7] N. Agafonova et al., OPERA Collaboration, JINST 4, P04018 (2009).

[8] J. Brunner, Phys. Lett. B731, 320 (2014).

[9] A. Fradette et al., Phys. Rev. D90, 035022 (2014).

[10] M. Drewes, et al., JHEP 1303, 096 (2013).

[11] M. Drewes, Int. J. Mod. Phys. E22, 1330019 (2013).

[12] T. Asaka and M. Shaposhnikov, Phys. Lett. B620, 17 (2005).

[13] M. Shaposhnikov, Nucl. Phys. B763, 49 (2007).

[14] L. Canetti et al., Phys. Rev. Lett. 110, 6, 061801 (2013).

[15] E. Bulbul et al., Astrophys. J. 789, 13 (2014).

[16] A. Boyarsky et al., Phys. Rev. Lett. 113, 251301 (2014).

[17] D. Gorbunov and M. Shaposhnikov, JHEP 0710, 015 (2007). Erratum-ibid. 1311, 101 (2013).

[18] L. Canetti and M. Shaposhnikov, JCAP 1009, 001 (2010).

[19] K. Kodama et al., DONUT Collaboration, Phys. Lett. B504, 218 (2001).

[20] K. Kodama et al., DONUT Collaboration, Phys. Rev. D78, 052002 (2008).

[21] N. Agafonova et al., OPERA Collaboration, Phys. Lett. B691, 138 (2010).

[22] N. Agafonova et al., OPERA Collaboration, JHEP 1311, 036 (2013).

[23] N. Agafonova et al., OPERA Collaboration, Phys. Rev. D89, 051102 (2014).

[24] N. Agafonova et al., OPERA Collaboration, PTEP 2014, 101C01 (2014). 
[25] N. Agafonova et al., OPERA Collaboration, Phys. Rev. Lett. 115, 121802 (2015).

[26] C. Lourenco, H.K. Wohri, Phys. Rept. 433, 127 (2006).

[27] J. Carvalho, Nuclear Physics A725, 269 (2003).

[28] H. Abramowicz, ZEUS Collaboration, JHEP 1309, 058 (2013).

[29] K.A. Olive et al. (Particle Data Group), Chin. Phys. C38, 090001 (2014).

[30] M. H. Reno, Phys. Rev. D 74, 033001 (2006).
[31] C. H. Albright and C. Jarlskog, Nucl. Phys. B 84, 467 (1975).

[32] G. De Lellis et al., Physics Reports 399, 227 (2004).

[33] A. Kayis-Topaksu et al., New J. Phys. 13, 093002 (2011).

[34] Babar and Belle Collaboration, Eur.Phys.J. C74, 3026 (2014).

[35] LHCb Collaboraiton, JHEP 1502, 121 (2015).

[36] C. Parkes et al., Nucl. Instrum. Meth. A604, 1 (2009). 